\title{
Projected future changes of tropical cyclone genesis frequency in the Northern Hemisphere based on a multi-timescale regression model
}

\section{jiuwei Zhao}

Nanjing University of Information Science and Technology

\section{Ruifen Zhan}

Fudan University https://orcid.org/0000-0001-7209-0904

\section{Yuqing Wang ( $\nabla$ yuqing@hawaii.edu )}

University of Hawaii at Manoa https://orcid.org/0000-0002-1691-8161

\section{Leishan Jiang}

University of Hawaii at Ma-noa

\section{Xin Huang}

Shanghai Typhoon Institute

\section{Article}

Keywords: tropical cyclone, genesis frequency, regression model

Posted Date: October 1st, 2021

DOI: https://doi.org/10.21203/rs.3.rs-951034/v1

License: (c) (i) This work is licensed under a Creative Commons Attribution 4.0 International License. Read Full License 


\section{Abstract}

Large uncertainties exist in the projected future TC genesis frequency (TCGF) due to the existence of various timescale internal climate variabilities and external forcing. Here, we introduce a statistical multitimescale TCGF regression model, including contributions by three interannual modes, two interdecadal modes, and a global warming mode. The model is shown to be able to capture well the present-day multitimescale changes in TCGF in the major TC basins in the Northern Hemisphere. The model results demonstrate that change in TCGF over the western North Pacific are predominantly modulated by internal climate variability while that over the eastern North Pacific is dominated by global warming and that over the North Atlantic is controlled about equally by the internal climate variability and global warming. Consistently, the model projects a significant increase over the eastern North Pacific and North Atlantic with insignificant trend over the western North Pacific.

\section{Background}

Numerous efforts have been made to understand tropical cyclone (TC) variability and to project their future changes owing to the large societal impacts from TCs ${ }^{1}$. In particular, understanding TC genesis is critically important but extraordinarily difficult ${ }^{2}$. TC genesis involves interactions among various timescale motions from large-scale circulation to small-scale convection. The large-scale environmental controls are largely modulated by not only local sea surface temperature (SST) modes but also transbasin SST anomalies related to internal (natural) climate modes (interannual and interdecadal/multidecadal variabilities) and long-term climate trends associated with external forcing (such as anthropogenic greenhouse gas emissions). The numerical modeling or dynamics downscaling projected either an increasing or a decreasing trend of TC genesis frequency (TCGF), remaining large uncertainty for future projections ${ }^{3-6}$. Therefore, understanding and projecting future TCGF changes is a topic of profound societal concerns and scientific interests

Considerable progress has been made in identifying and understanding large-scale factors that affect the variability of TCGF in the past decades. On the interannual timescale, the El Niño-Southern Oscillation (ENSO) has a pantropic impact on TCGFs over the western North Pacific (WNP) ${ }^{7}$, the eastern North Pacific (ENP) ${ }^{8,9}$ and the North Atlantic $(N A)^{10}$. During the warm phase of ENSO, TCGF over the ENP increases mainly due to the favorable atmospheric conditions and large oceanic energy supply, while TCGF over the NA is significantly suppressed due to the increased vertical wind shear and negative lowlevel vorticity anomalies, indicating a significant interannual seesaw across basins. Over the WNP, the canonical ENSO tends to induce a dipole response of TCGF change between the southeastern and northwestern quadrants of the basin ${ }^{7}$. WNP TCGF is also linked to the SST gradient between the Southwest Pacific and the western Pacific warm pool ${ }^{11}$, and the SST anomalies (SSTAs) over the eastern Indian Ocean (EIO) ${ }^{17}$ and the tropical NA (TNA) ${ }^{18}$. Moreover, the inter-basin SST gradient (also called "relative" SST) between the ENP and the NA affects TCGFs over the ENP and the NA ${ }^{14-16}$. 
TCGF also exhibits strong decadal/interdecadal or multidecadal variabilities ${ }^{17-21}$. Many studies have shown that the Atlantic Multidecadal Oscillation (AMO) ${ }^{24}$ affects TCGFs over the NA, ENP and WNP by modulating the local and remote atmospheric circulations. Besides the AMO, the Pacific Decadal Oscillation (PDO) or Interdecadal Pacific Oscillation (IPO) ${ }^{22,26}$ plays an important role in modulating TCGFs over the North Pacific with a basin-wide uniform increase in its positive phase and a decrease in its negative phase ${ }^{18,21-23}$. The TCGFs over the NA and North Pacific also exhibit a seesaw due to the trans-basin effects of SST on decadal timescale $22,24-26$.

In addition to internal climate variability, anthropogenic green-house-gas-induced warming can also influence TCGF ${ }^{6}$. However, how and to what extent the green-house-gas-induced GW affects TCGF remain uncertain. Most climate models projected a future decrease, while some other studies projected an increase in global TCGF $3,5,27$. Such opposing results call for caution in the interpretation of existing climate simulations and the confidence in the projected TCGF change by climate models ${ }^{28}$. Uncertaintied in the SST warming pattern and model biases are important factors that can lead to a large diversity in the projected future changes in TCGF ${ }^{29-31}$.

The TC genesis potential index (GPI) is commonly used to explore TCGF in the present-day and future climates. Gray ${ }^{32}$ first constructed a seasonal genesis parameter based on several environmental variables, which was further modified and reconstructed in many other studies ${ }^{33-37}$. Among them, the GPI developed by Emanuel and Nolan ${ }^{33}$ has been widely used with skills in replicating the seasonal and interannual variabilities of the observed TCGF in various TC basins ${ }^{38}$. However, the GPI performance is not always good in either reproducing the long-term variability or projecting future changes in $\mathrm{TCGF}^{35}$.

It is well known that the SST not only affects the atmospheric and oceanic thermostat, but also modulates large-scale dynamical circulation both locally and remotely. Therefore, in this study, we introduce a multi-timescale regression model of TCGF based on five key SST factors and a GW factor, all derived from SST data. We will show that the new model can reproduce the observed multi-timescale variability in TCGF in the Northern Hemisphere $(\mathrm{NH})$ in the present-day climate and can well delineate the observed TCGF changes on interannual and interdecadal timescales as well as the long-term trend. The new model is also used to project future changes in TCGF in the three main TC basins, namely the WNP, ENP, and NA, in the NH with the future SST pattern and key SST factors constructed based partly on the historical data and partly on the state-of-the-art climate model projections, including the GW trend. The results show a steady future increase in TCGF over both the ENP and the NA with insignificant trends over the NWP.

\section{Results}

Figure 1 shows the feedback coefficients of the responses of TCGF to the six individual key factors (Supplementary Figs. S1 and S2, See method) based on the generalized equilibrium feedback assessment (GEFA) method ${ }^{39,40}$ (See method). A positive (negative) response means that the TCGF is 
above (below) normal when the factor has a positive (negative) anomaly. It is obvious that all six factors have significant influence on TCGFs over the three TC basins in the $\mathrm{NH}$, but exhibit different spatial patterns, which are consistent with previous studies as introduced above. These significant influences can be explained by the responses of the large-scale environmental conditions to these factors (Supplementary Figs. S3 and S4). Namely, the large-scale environmental atmospheric anomalies induced by these factors provide either favorable or unfavorable conditions for TC genesis on multi-timescales over different basins in the $\mathrm{NH}$.

The response coefficients also reflect the relative contributions of individual factors to the TCGF. Over the NA, the TNA, AMO and GW contribute the most to TCGF, and the ENSO and EIO contribute the second most. Over the ENP, GW and AMO contributes the most, and ENSO contribute the second most, with significant negative (positive) contributions over the eastern (western) ENP. Over the WNP, ENSO contributes the most with opposite signs over the northwestern and southeastern quadrants, and AMO and GW contribute the second most. AMO seems to be the most important factor for TCGF over the South China Sea. These results consistently show that the internal climate variability is more important for TCGF over the WNP than the GW although the GW also exerts significant impact, while the GW dominates other factors over the ENP and the internal variability and the GW are about equally important over the NA.

\section{Performance of the regression model in reproducing the multi-timescale variability of TCGF}

Here, the performance of the regression model in reproducing the multi-timescale variability of TCGF in the $\mathrm{NH}$ is evaluated and the estimated TCGF is compared with the commonly used GPI as well as the state-of-the-art climate models. Figure 2 compares the climatological means of the observed TCGF (Fig. 2a), the leave-one-out regressed TCGF (Fig. 2b), which is independent of the withholding samples, and the commonly used GPI (Fig. 2C), all averaged during JJASON for the period 1960-2019 in the NH. Overall, the spatial pattern correlation between the observed and regressed TCGFs is higher than that between the observed TCGF and the commonly used GPI. Specifically, the GPI shows considerable discrepancies in both the amplitude and spatial distribution of the climatological TCGF (Fig. 2c). In sharp contrast, the regressed TCGF shows good consistency with the observed climatological TCGF, with the spatial correlation with the observed as high as 0.99 (Fig. 2b).

We further examined how skillful the new model is in reproducing the observed TCGFs in the three NH TC basins. Figures $2 \mathrm{~d}-2 \mathrm{f}$ show the correlation coefficients of the observed TCGF with the regressed TCGF and the commonly used GPI in the 1960-2019 period. The red bars represent the correlation coefficients calculated for the raw time series while the blue bars for the components beyond the interannual time scale. In general, the GPI fails to reproduce the multi-timescale variations of the observed TCGF over the ENP and WNP (red bars), especially for the long-term variations (blue bars). The correlation between the GPI based on the NCEP reanalysis and the observed TCGF is weak over three basins, with the correlation coefficients being, respectively, 0.18 over the NA, 0.33 ENP, and -0.16 over the WNP. The correlations are $0.6,0.37$ and -0.16 based on the ERA 5 reanalysis, respectively. In sharp contrast, the correlation 
coefficients between the regressed and the observed TCGFs reach $0.63,0.48$ and 0.34 , respectively, over the NA, the ENP, and the WNP, all being statistically significant over $95 \%$ confidence level (Fig. $2 \mathrm{~d}$-f). More importantly, after we filtered out the interannual variability, we found that the regressed TCGF well captured the long-term variability of the observed TCGF with the correlation coefficients reaching 0.87 , 0.74 and 0.48 over the NA, ENP and WNP, respectively, all being statistically significant over $95 \%$ confidence level (blue bar in Fig. 2d-f). However, the correlations between the observed TCGF and the GPI are insignificant except for over the NA for the ERA data and even for all of three basins for the NCEP data. Furthermore, for both reanalysis datasets, the long-term variations between the GPI and the observed TCGF are negatively correlated over the WNP (Fig. 2f). This strongly suggests that the regressed model developed in this study can better represent the multi-scale changes in TCGF in the three NH TC basins, especially for the long-term changes, compared to the commonly used GPI.

Currently, the state-of-the-art climate models are widely used in studying the variability and future change in TCGF 5,6 . We examined the performance of the European Center for Medium Weather Forecasting's Integrated Forecasting System (ECMWF-IFS) with a horizontal resolution of $0.5^{\circ} \times 0.5^{\circ}$ in reproducing the multi-timescale variability of TCGF during 1960-2014 (Supplementary Fig. S5). The ECMWF-IFS is one of the models participating the CMIP6 High Resolution Model Intercomparison Project (HighResMIP) ${ }^{41}$. The model reproduces the TCGF variability in response to ENSO, EIO and TNA SST anomalies quite well. However, the model produces the opposite decadal variation of TCGF over the ENP and the opposite longterm trends in the observed TCGF over both the WNP and the ENP. We also examined the performances of several other models from the HighResMIP, including the EC-Earth, MRI-ESM, FGOALS, HadGEM3-GC3 and NICAM, and the results (Figure not shown) are similar to those from the ECMWF-IFS. This implies that most of the state-of-the-art high-resolution models from the CMIP6 still have low still in representing the TCGF change beyond the interannual timescale.

To understand why our multi-timescale regression model is skillful in reproducing the observed TCGF, we analyzed the connection of the estimated TCGF and GPI with the six key factors used in the regression model. Figure 3 shows the composite differences in the observed TCGF, the regressed TCGF, and GPI between the positive and negative phases of the first five factors, and their trend increments as the GW impact. To confirm the data independency, the composite differences in GPI based on the ERA5 reanalysis data are shown in SI Appendix Fig. S6. As expected, the regressed TCGF well matches the responses of the observed TCGF anomalies to the six key factors over the three TC basins, with the spatial distribution consistent with that of the observed TCGF. Since these key factors largely control the TCGF change from interannual and interdecadal variations to long-term trend and also include both intrabasin and inter-basin influences, the regression model can well reproduce the multi-timescale variability of the observed TCGF over the major TC basins in the NH. In contrast, the commonly used GPI based on the two reanalysis datasets shows large discrepancies from the observed TCGF. The largest discrepancy is found in the responses to both the interdecadal factors and the GW mode, giving rise to nearly opposite signs between the GPI and the observed TCGF in the three basins. Although the GPI reproduces the observed TCGF responses to the interannual factors reasonably well, discrepancies still exist in the 
extreme values and their spatial locations. These results are consistent with those found in previous studies based on observations and climate models ${ }^{35,42}$ and are also confirmed by comparing the correlation coefficients between these key factors and, respectively, the observed TCGF, the regressed TCGF, and the commonly used GPI (SI Appendix Table S1).

\section{Projected future change of TCGF in the NH using the multi-timescale regression model}

Since the regression model can well capture the long-term changes in the observed TCGF in the NH, if the future changes in the six key factors are known, it can be used to project future changes in TCGF in the $\mathrm{NH}$. For example, the future changes in the six key factors can be obtained from global model ensemble simulations of the CMIP5 or CMIP6. However, as mentioned above, climate models from the CMIP6 HighResMIP have low skills in reproducing the responses of the TCGF to long-term climate changes. In this study, the future changes in the six key factors during 2019-2069 are constructed based partly on the historical data and partly on the model projections. For each member among the 100 ensemble members, the indices of those interannual factors are created by randomly picking up the corresponding interannual indices from the historical data during 1960-2019 and repeating 100 times to generate 100 ensemble members. Namely, there are 10,000 samples in total. We constructed the time series of the IPO and the AMO during 2019-2069 based on the 100 ensemble mean of the Max Planck Institute Earth System Model (MPI-ESM). Consistent with previous studies ${ }^{43-45}$, the decadal/interdecadal factors, namely the IPO and the AMO, are the second principal components (PCs) from the empirical orthogonal function (EOF) analysis of the filtered SST data over the respective Pacific and North Atlantic regions (Supplementary Fig. S7) for respective RCP2.6 (low warming condition), RCP4.5 (medium warming condition), and RCP8.5 (extreme warming condition) scenarios. Similarly, the GW mode during 2019-2069 was obtained by the GMSST in RCP2.6, RCP4.5 and RCP 8.5 scenarios (Supplementary Fig. S8), respectively.

Figure 4 shows the projected TCGF changes over the three main TC basins in the NH during 2019-2069 based on RCP2.6, RCP4.5 and RCP8.5 scenarios using the regression model. For the NA (Figs. 4a, 4d and $4 \mathrm{~g}$ ), the probability of significant linear trends in TCGF is weak under the low warming condition with the probability of only $34.97 \%$, while it becomes significant in both the medium and extreme warming conditions and even reaches $100 \%$ in an extreme warming condition. This means that the TCGF over the NA is largely contributed by the internal climate modes such as the AMO and IPO and external forcing such as GW, and the GW is a dominant influencing factor under extreme warming condition. For the ENP (Figs. 4b, 4e and 4h), the probability of significant increasing trends in TCGF under the slow warming condition is the highest among the three NH TC basins with the probability of about $70 \%$ (Fig. 4b), while the probability reaches $100 \%$ in both the medium and extreme warming conditions. Over the WNP (Figs. 4c, $4 \mathrm{f}$ and 4i), the probabilities of significant linear trend in TCGF from 2019-2069 are considerably small in all the three warming conditions, and the trend is generally not significant. This strongly suggests that there would be more TCs to form over the ENP and the NA under GW with higher confidence for the ENP, while the future change in TCGF over the WNP is strongly modulated by the internal climate variability. 
The projected increase in TCGF in the ENP and NA is consistent with some previously reported downscaling results ${ }^{3,27}$, but is different from the projected decrease by the majority of climate models ${ }^{5}$. The climate model projected decrease is ranked as being low-to-medium confidence, even lower for individual TC basins. This is because the state-of-the-art climate models have very limited skill in representing the TCGF change beyond the interannual timescale ${ }^{28,46}$. In addition, our results also strongly suggest that the impact of GW on TCGF is predominant over the ENP and NA, but is insignificant over the WNP, where the internal climate variability may play dominant roles in controlling the long-term changes in TCGF, as also indicated in some previous studies ${ }^{23,30}$.

\section{Conclusions And Discussion}

In this study, a multi-timescale TCGF regression model has been constructed with six key climate factors that significantly control TCGF at different timescales in the $\mathrm{NH}$. The regression model is skillful in reproducing the multi-timescale variability of TCGF over the major TC basins in the $\mathrm{NH}$. Using this model and the projected key factors under three assumed GW scenarios, we have projected a steady increase in TCGF over the ENP and NA in a warmer climate but insignificant trend over the WNP, where the future long-term changes in TCGF are dominated by internal climate (mainly the interdecadal) variability.

In addition to the six well-documented key factors used in the regression model, previous studies have also revealed the significant impacts of other factors on TCGF in the $\mathrm{NH}$, such as the Pacific Meridional Mode and the inter-hemispheric SST gradient ${ }^{11,47}$. Nevertheless, their impacts could have been partially included in the six key factors. This may be the reason why the regression model with the six key factors is skillful in reproducing the multi-timescale variability of the observed TCGF. To project the future TCGF change, some assumptions have been made, including the interannual variations remained under the present-day climate and reasonable simulations of decadal/interdecadal SST modes under assumed GW scenarios by state-of-the-art climate models. In addition, the possible nonlinear interactions among motions on various timescales in our projections of the key factors are not considered and are assumed quite weak. Nevertheless, the projected future TCGF in this study supports the downscaling results in several previous studies ${ }^{3,27}$. Therefore, the newly developed multi-timescale regression model can provide an additional reference with an alternative independent approach.

\section{Methods}

\section{Spatial distribution of TCGF}

Inspired by the kernel density method of $\mathrm{TCGF}^{48}$, the $\mathrm{NH}$ domain is meshed into $1^{\circ} 1^{\circ} \mathrm{grid}$ box and then the TCGF in each grid box is counted as the sum of the TCs formed in an area of 10 degrees in the zonal direction and 5 degrees in the meridional direction centred at this grid box. This method, as a smoother, can not only reduce the possible uncertainty due to the scattering nature of TC genesis or bias of genesis location in the TC data, but also establish a stable relationship between the observed TCGF and the related environmental factors. We chose a $20^{\circ} 10^{\circ}$ domain considering the fact that the synoptic waves, 
such as equatorial Rossby waves, mixed-Rossby-gravity waves, and easterly waves, or other types of synoptic disturbances, that trigger TC genesis have horizontal scale of about two thousands of kilometers in the zonal direction and e-folded in the meridional direction ${ }^{49}$. However, we also tested $30^{\circ} 15^{\circ}$ and $10^{\circ} 5^{\circ}$ domains. Basically, the result from $30^{\circ} 15^{\circ}$ domain shows a similar correlation with that using $20^{\circ} 10^{\circ}$ domain. However, the climatological TC genesis density over the WNP is higher than that over the ENP due to the large TC-counting domain, which is unrealistic. The result from $10^{\circ} 5^{\circ}$ domain is too chaotic owing to the small sample size. We focus on TCGF during June-November (JJASON) in the $\mathrm{NH}$ where about $85 \%$ of global TCs form each year ${ }^{50}$. Note that since TCs over the North Indian Ocean mainly form in the pre-monsoon (April and May) and the post-monsoon (October and November) seasons ${ }^{51}$, the TCGF over the North Indian Ocean is not included in this study. Therefore, this study has focused on TCGFs over the three main TC basins in the NH, including the WNP, the ENP, and the NA.

\section{Six oceanic factors defined by SST}

The SST data are used to define the key SST indices controlling the interannual variability of TCGFs, including ENSO, EIO and TNA indices with the decadal signal ( $\geq 10$ years) removed based on the fast Fourier transform. Here, we define the SST in JJASON averaged over the Nino3.4 ( $\left.5^{\circ} \mathrm{S}-5^{\circ} \mathrm{N}, 120^{\circ} \mathrm{W}-170^{\circ} \mathrm{W}\right)$ as the ENSO index, that averaged over the EIO $\left(10^{\circ} \mathrm{S}-22.5^{\circ} \mathrm{N}, 75^{\circ} \mathrm{E}-100^{\circ} \mathrm{E}\right)$ as the $\mathrm{EIO}$ index ${ }^{12}$, and that averaged over the tropical Atlantic $\left(5^{\circ} \mathrm{N}-25^{\circ} \mathrm{N} ; 30^{\circ} \mathrm{W}-70^{\circ} \mathrm{W}\right)$ as the TNA index ${ }^{13}$. All the ENSO, EIO and TNA warm and cold events are selected based on the criteria of above and below 0.7 standard deviations, respectively. Based on these criteria, 20 ENSO events, including 9 El Niño and 11 La Niña, 23 ElO events, including 10 warm and 13 cold episodes, and 20 TNA events, including 10 warm and 10 cold events are identified (Supplementary Figure S1). Since both the IPO and AMO indices mainly control the variabilities of TCGFs with timescales beyond the interannual timescale, the interannual variability ( $<10$ years) is removed from both the IPO and AMO indices using the fast Fourier transform. The IPO experienced two negative phases (1960-1975 and 1998-2019) and one positive phase (1976-1997), while the AMO exhibited one negative phase (1960-1995) and one positive phase (1996-2019) in our study period. The global warming time series is defined by the global mean SST over $45^{\circ} \mathrm{S}-45^{\circ} \mathrm{N}, 0^{\circ}-360^{\circ}$.

\section{Construction of a multi-timescale TCGF regression model in the $\mathrm{NH}$}

$$
T C G F=a * E N S O+b * E I O+c * T N A+d * A M O+e * I P O+f * G M S S T+\text { const, (1) }
$$


The new multi-timescale regression model developed in this study predicts the TCGF in the NH with the factors of both internal climate variability and external forcing and is expressed in the form. We consider the terms on the RHS of Eq. (1) as being linearly proportional to six key factors controlling the TCGF in the $\mathrm{NH}$ with five being related to the internal climate variability and one being the external forcing. Among the internal climate factors, there are three interannual climate modes (ENSO, EIO SST, and the TNA SST) and two interdecadal climate modes (the IPO and the AMO). The external forcing (GWSST) is represented by the increasing global mean SST averaged over $45^{\circ} \mathrm{S}-45^{\circ} \mathrm{N}, 0^{\circ}-360^{\circ}$. These factors have been recognized to greatly modulate the interannual and longer-timescale variabilities of TCGF. The regressed coefficients in the regression model Eq. (1) vary with basin as inferred in Table 1. The normalized time series of these factors are shown in Supplementary Fig. S1, and the composite difference in SST anomalies between the positive and negative phases of each factor is displayed in Supplementary Fig. S2. The SST anomalies also show a significant GW pattern in response to the increasing $\mathrm{CO}_{2}$ concentration (Supplementary Fig. S2f). It is noted that the SST warming rate is different over different basins, suggesting that the GW impact on TCGF could differ across basins. To confirm the significance of these six factors, we examined the relationships between the observed TCGF and these factors and the possibly involved physical mechanisms.

\section{The GPI and generalized equilibrium feedback assessment (GEFA)}

The performance of the newly constructed multi-timescale regression model is compared with the widely used GPI developed by Emanuel and Nolan ${ }^{33}$, which is a function of several environmental variables given below:

$$
G P I=\left|10^{5} \eta\right|^{\frac{3}{2}}\left(\frac{R h u m}{50}\right)^{3}\left(\frac{V_{\text {pot }}}{70}\right)^{3}(1+0.1 V W S)^{-2},
$$

where is the absolute vertical vorticity at $850 \mathrm{hPa}$, Rhum is the relative humidity at $600 \mathrm{hPa}$, is the TC maximum potential intensity ${ }^{52}$, and VWS is the vertical shear of horizontal winds between 200 and 850 $\mathrm{hPa}$.

The newly constructed multi-timescale regression model describes the relationship between the TCGF averaged over a TC season and the key factors that are known to control different timescale variabilities of TCGF and thus can reproduce the TCGF in each grid box in the NH. To confirm the validity of the multitimescale regression model, the leave-one-out cross-validation method ${ }^{53}$ is used to hindcast the observed TCGF during 1960-2019. The generalized equilibrium feedback assessment (GEFA) ${ }^{39,40}$ is conducted to evaluate the relative contributions of individual key factors to the observed TCGF variability. With the promising skill in reproducing the observed present-day variability of TCGF, the multi-timescale regression 
model is then used to project future changes in TCGF in the three major TC basins in the NH with the projected key factors described in detail in Results.

\section{Time series of IPO, AMO and GMSST from MPI-ESM}

The projected SST data (RCP2.6, RCP4.5 and RCP8.5) from the Max Planck Institute (MPI) contain 100 ensemble members, covering the period 2006-2099. We first removed the high-frequency signal from the June-November averaged SST data and then conducted the empirical orthogonal function (EOF) analysis over the NA $\left(0^{\circ}-70^{\circ} \mathrm{N}, 0^{\circ}-80^{\circ} \mathrm{W}\right)$ and Pacific $\left(45^{\circ} \mathrm{S}-45^{\circ} \mathrm{N}, 100^{\circ} \mathrm{E}-60^{\circ} \mathrm{W}\right)$ regions as shown in

Supplementary Fig. S7. According to previous studies ${ }^{43-45}$, the IPO and AMO time series can be represented by the second EOF modes. The spatial patterns of the IPO and AMO in Supplementary Fig. S7 are averaged based on their corresponding positive phases. The GW time series are derived from MPIESM. The GMSST anomalies are obtained by removing the ensemble mean SST in 2019 and we normalized the GMSST from 2019-2099 by dividing the standard deviation of GMSST from 1960-2019 using observational data and adding the value of observed GMSST in 2019 (Fig. S8).

\section{Statistics}

In this study, the statistical significances were checked based on three methods: the two-tailed Student's t test, the two-sided Mann-Kendall trend test and the Monte-Carlo test. The Student's t test is adopted for testing if the composites of TCTD and circulation fields are significant or not. The degree of freedom is 58 for the 60-year data. The Mann-Kendall trend test is used to verify if the linear trends of projecting and simulating TCGF with the degree of freedom 60 for historical data and 50 for future projection results, are significant or not. The Monte-Carlo test repeated the sampling randomly for 100 times, and tested whether the GEFA contribution is significant above $90 \%$ confidence level.

\section{Data availability}

The 6-hourly NH TC best-track dataset is obtained from the International Best Track Archive for Climate Stewardship version 4 (IBTrACS v4; https://www.ncei.noaa.gov/data/international-best-track-archive-forclimate-stewardship-ibtracs/v04r00/access/netcdf/), which includes the central position (longitude and latitude) and the intensity in terms of maximum sustained near-surface wind speed of each TC during the 1960-2019 period. The TC genesis location is defined as the central position where a TC reaches 35 knots for the first time.

The monthly Extended Reconstructed SST version 5 data ${ }^{54}$ with a horizontal resolution of $2^{\circ} 2^{\circ}$ were obtained from the National Oceanic and Atmospheric Administration (NOAA). In the history data, the IPO index (https://www.esrl.noaa.gov/psd/data/timeseries/IPOTPI/) and AMO index (https://www.esrl.noaa.gov/psd/data/ timeseries/AMO/) were downloaded from the Earth System Research Laboratory (ESRL) of NOAA, both were constructed based on the same monthly SST dataset. 
The atmospheric data, including winds and relative humidity, were obtained from the National Centres for Environmental Prediction/National Centers for Atmospheric Research (NCEP/NCAR) reanalysis I with a horizontal resolution of $2.5^{\circ} 2.5^{\circ}$ ( https://psl.noaa.gov/data/gridded/data.ncep.reanalysis. html) ${ }^{56}$. The HighResMIP CMIP6 data for ECMWF, EC_Earth, MRI, HadGEM and NICAM were downloaded from http://data.ceda.ac.uk/badc/cmip6/data/CMIP6/HighResMIP, and those for FGOALS were provided by State Key Laboratory of Numerical Modeling for Atmospheric Sciences and Geophysical Fluid Dynamics of Institute of Atmospheric Physics, which were downloaded from https://figshare.com/s/658ac25c74735730ac5b. The model-projected SST data were from the Max Planck Institute Earth System Model (MPI-ESM), which contain 100 ensemble members covering the period 2006-2099 and can be obtained on personal request (https://esgf-data.dkrz.de/projects/mpi-ge/)

\section{Declarations}

\section{Acknowledgments.}

This work has been supported by the Guangdong Major Project of Basic and Applied Basic Research (2020B0301030004) and the National Natural Science Foundation of China (grants 4210050250 , 42075015, 41875114).

\section{Author Contributions}

J. Z and R. Z conceived the idea and designed this study in discussion with Y. W., L. J and X. H conceived part of this work. J. Z and R. Z analyzed the data and generated the plots. J. Z, R. Z, Y. W. discussed the results and jointly contributed to writing the manuscript. All authors approved the final version of the manuscript.

Competing interest Statement: We declare no competing interests.

\section{References}

1. Walsh, K. J. E. et al. Hurricanes and Climate: The U.S. CLIVAR Working Group on Hurricanes. Bull. Am. Meteorol. Soc. 96, 997-1017 (2015).

2. Emanuel, K. 100 Years of Progress in Tropical Cyclone Research. Meteorol. Monogr. 59, 15.1-15.68 (2018).

3. Emanuel, K. A. Downscaling CMIP5 climate models shows increased tropical cyclone activity over the 21st century. Proc. Natl. Acad. Sci. 110, 12219-12224 (2013).

4. Knutson, T. R. et al. Dynamical Downscaling Projections of Twenty-First-Century Atlantic Hurricane Activity: CMIP3 and CMIP5 Model-Based Scenarios. J. Clim. 26, 6591-6617 (2013). 
5. Knutson, T. et al. Tropical Cyclones and Climate Change Assessment: Part II: Projected Response to Anthropogenic Warming. Bull. Am. Meteorol. Soc. 101, E303-E322 (2020).

6. Knutson, T. et al. Tropical Cyclones and Climate Change Assessment: Part I. Detection and Attribution. Bull. Am. Meteorol. Soc. BAMS-D-18-0189.1 (2019) doi:10.1175/BAMS-D-18-0189.1.

7. Wang, B. \& Chan, J. C. L. How Strong ENSO Events Affect Tropical Storm Activity over the Western North Pacific*. J. Clim. 15, 1643-1658 (2002).

8. Jin, F.-F., Boucharel, J. \& Lin, I.-I. Eastern Pacific tropical cyclones intensified by El Niño delivery of subsurface ocean heat. Nature $516,82-85$ (2014).

9. Patricola, C. M., Chang, P. \& Saravanan, R. Degree of simulated suppression of Atlantic tropical cyclones modulated by flavour of El Niño. Nat. Geosci. 9, 155-160 (2016).

10. Wang, H. et al. How Well Do Global Climate Models Simulate the Variability of Atlantic Tropical Cyclones Associated with ENSO? J. Clim. 27, 5673-5692 (2014).

11. Zhan, R., Wang, Y. \& Wen, M. The SST Gradient between the Southwestern Pacific and the Western Pacific Warm Pool: A New Factor Controlling the Northwestern Pacific Tropical Cyclone Genesis Frequency. J. Clim. 26, 2408-2415 (2013).

12. Zhan, R., Wang, Y. \& Lei, X. Contributions of ENSO and East Indian Ocean SSTA to the Interannual Variability of Northwest Pacific Tropical Cyclone Frequency*. J. Clim. 24, 509-521 (2011).

13. Yu, J., Li, T., Tan, Z. \& Zhu, Z. Effects of tropical North Atlantic SST on tropical cyclone genesis in the western North Pacific. Clim. Dyn. 46, 865-877 (2016).

14. Vecchi, G. A. \& Soden, B. J. Effect of remote sea surface temperature change on tropical cyclone potential intensity. Nature 450, 1066-1070 (2007).

15. Johnson, N. C. \& Xie, S.-P. Changes in the sea surface temperature threshold for tropical convection. Nat. Geosci. 3, 842-845 (2010).

16. Mei, W., Kamae, Y., Xie, S.-P. \& Yoshida, K. Variability and Predictability of North Atlantic Hurricane Frequency in a Large Ensemble of High-Resolution Atmospheric Simulations. J. Clim. 32, 3153-3167 (2019).

17. He, H. et al. Decadal changes in tropical cyclone activity over the western North Pacific in the late 1990s. Clim. Dyn. 45, 3317-3329 (2015).

18. Zhao, J., Zhan, R., Wang, Y. \& Xu, H. Contribution of Interdecadal Pacific Oscillation to the recent abrupt decrease in tropical cyclone genesis frequency over the western North Pacific since 1998. J. Clim. (2018) doi:10.1175/JCLI-D-18-0202.1. 
19. Zhao, J., Zhan, R. \& Wang, Y. Global warming hiatus contributed to the increased occurrence of intense tropical cyclones in the coastal regions along East Asia. Sci. Rep. 8, (2018).

20. Zhang, W. et al. Dominant Role of Atlantic Multidecadal Oscillation in the Recent Decadal Changes in Western North Pacific Tropical Cyclone Activity. Geophys. Res. Lett. (2018) doi:10.1002/2017GL076397.

21. Chan, J. C. L. Decadal variations of intense typhoon occurrence in the western North Pacific. Proc. R. Soc. Math. Phys. Eng. Sci. 464, 249-272 (2008).

22. Li, W., Li, L. \& Deng, Y. Impact of the Interdecadal Pacific Oscillation on Tropical Cyclone Activity in the North Atlantic and Eastern North Pacific. Sci. Rep. 5, (2015).

23. Zhao, J., Zhan, R., Wang, Y., Xie, S.-P. \& Wu, Q. Untangling impacts of global warming and Interdecadal Pacific Oscillation on long-term variability of North Pacific tropical cyclone track density. Sci. Adv. 6, eaba6813 (2020).

24. Dong, B. \& Dai, A. The influence of the Interdecadal Pacific Oscillation on Temperature and Precipitation over the Globe. Clim. Dyn. 45, 2667-2681 (2015).

25. Dong, L. et al. The Footprint of the Inter-decadal Pacific Oscillation in Indian Ocean Sea Surface Temperatures. Sci. Rep. 6, (2016).

26. Huang, Y., Wu, B., Li, T., Zhou, T. \& Liu, B. Interdecadal Indian Ocean Basin Mode Driven by Interdecadal Pacific Oscillation: A Season-Dependent Growth Mechanism. J. Clim. 32, 2057-2073 (2019).

27. Park, D.-S. R. et al. Asymmetric response of tropical cyclone activity to global warming over the North Atlantic and western North Pacific from CMIP5 model projections. Sci. Rep. 7, 41354 (2017).

28. Roberts, M. J. et al. Impact of Model Resolution on Tropical Cyclone Simulation Using the HighResMIP-PRIMAVERA Multimodel Ensemble. J. Clim. 33, 2557-2583 (2020).

29. Knutson, T. R. et al. Global Projections of Intense Tropical Cyclone Activity for the Late TwentyFirst Century from Dynamical Downscaling of CMIP5/RCP4.5 Scenarios. J. Clim. 28, 7203-7224 (2015).

30. Murakami, H. et al. Detected climatic change in global distribution of tropical cyclones. Proc. Natl. Acad. Sci. 117, 10706-10714 (2020).

31. Zhao, J., Zhan, R. \& Wang, Y. Different responses of tropical cyclone tracks over the western North Pacific and North Atlantic to two distinct SST warming patterns. Geophys. Res. Lett. e2019GL086923 (2020) doi:10.1029/2019GL086923.

32. Gray, B. Tropical cyclone genesis. 
33. Emanuel, K. \& D. S. Nolan. Tropical cyclone activity and global climate. (2004).

34. Emanuel, K. Tropical cyclone activity downscaled from NOAA-CIRES Reanalysis, 1908-1958. J. Adv. Model. Earth Syst. 2, 1 (2010).

35. Bruyère, C. L., Holland, G. J. \& Towler, E. Investigating the Use of a Genesis Potential Index for Tropical Cyclones in the North Atlantic Basin. J. Clim. 25, 8611-8626 (2012).

36. Zhang, L., Karnauskas, K. B., Donnelly, J. P. \& Emanuel, K. Response of the North Pacific Tropical Cyclone Climatology to Global Warming: Application of Dynamical Downscaling to CMIP5 Models. J. Clim. 30, 1233-1243 (2017).

37. Wang, B. \& Moon, J.-Y. An Anomalous Genesis Potential Index for MJO Modulation of Tropical Cyclones. J. Clim. 30, 4021-4035 (2017).

38. Camargo, S. J., Emanuel, K. A. \& Sobel, A. H. Use of a Genesis Potential Index to Diagnose ENSO Effects on Tropical Cyclone Genesis. J. Clim. 20, 4819-4834 (2007).

39. Liu, Z. \& Wu, L. Atmospheric Response to North Pacific SST: The Role ofOcean-Atmosphere Coupling. J. Clim. 17, 1859-1882 (2004).

40. Liu, Z., Wen, N. \& Liu, Y. On the Assessment of Nonlocal Climate Feedback. Part I: The Generalized Equilibrium Feedback Assessment. J. Clim. 21, 134-148 (2008).

41. Haarsma, R. J. et al. High Resolution Model Intercomparison Project (HighResMIP v1.0) for CMIP6. Geosci. Model Dev. 9, 4185-4208 (2016).

42. Yokoi, S., Takayabu, Y. N. \& Chan, J. C. L. Tropical cyclone genesis frequency over the western North Pacific simulated in medium-resolution coupled general circulation models. Clim. Dyn. 33, 665-683 (2009).

43. Henley, B. J. et al. A Tripole Index for the Interdecadal Pacific Oscillation. Clim. Dyn. 45, 30773090 (2015).

44. Yang, S. et al. El Niño-Southern Oscillation and its impact in the changing climate. Natl. Sci. Rev. 5, 840-857 (2018).

45. Guan, B. \& Nigam, S. Analysis of Atlantic SST Variability Factoring Interbasin Links and the Secular Trend: Clarified Structure of the Atlantic Multidecadal Oscillation. J. Clim. 22, 4228-4240 (2009).

46. Roberts, M. J. et al. Projected Future Changes in Tropical Cyclones Using the CMIP6 HighResMIP Multimodel Ensemble. Geophys. Res. Lett. 47, (2020).

47. Zhang, W., Vecchi, G. A., Murakami, H., Villarini, G. \& Jia, L. The Pacific Meridional Mode and the Occurrence of Tropical Cyclones in the Western North Pacific. J. Clim. 29, 381-398 (2016). 
48. Lu, M. \& Xiong, R. Spatiotemporal Profiling of Tropical Cyclones Genesis and Favorable Environmental Conditions in the Western Pacific Basin. Geophys. Res. Lett. 46, 11548-11558 (2019).

49. Chen, G. \& Huang, R. Interannual Variations in Mixed Rossby-Gravity Waves and Their Impacts on Tropical Cyclogenesis over the Western North Pacific. J. Clim. 22, 535-549 (2009).

50. Murakami, H. et al. Simulation and Prediction of Category 4 and 5 Hurricanes in the HighResolution GFDL HiFLOR Coupled Climate Model. J. Clim. 28, 9058-9079 (2015).

51. Chen, X., Wang, Y. \& Zhao, K. Synoptic Flow Patterns and Large-Scale Characteristics Associated with Rapidly Intensifying Tropical Cyclones in the South China Sea. Mon. Weather Rev. 143, 64-87 (2015).

52. Emanuel, K. A. The Maximum Intensity of Hurricanes. J. Atmospheric Sci. 45, 1143-1155 (1988).

53. Molinaro, A. M., Simon, R. \& Pfeiffer, R. M. Prediction error estimation: a comparison of resampling methods. Bioinformatics 21, 3301-3307 (2005).

54. Huang, B. et al. Extended Reconstructed Sea Surface Temperature, Version 5 (ERSSTv5): Upgrades, Validations, and Intercomparisons. J. Clim. 30, 8179-8205 (2017).

55. Smith, T. M. \& Reynolds, R. W. Improved Extended Reconstruction of SST (1854-1997). J. Clim. $17,2466-2477$ (2004).

56. Kalnay, E. et al. The NCEP/NCAR 40-Year Reanalysis Project. Bull. Am. Meteorol. Soc.77, 437-471 (1996).

\section{Table 1}

Table 1: The corresponding coefficients of the six factors (predictors) in Eq. (1) over the three main TC basins in the NH. $a-f$ are coefficients of ENSO, EIO SST, TNA SST, IPO, AMO and GW, respectively.

\begin{tabular}{|lllllll|}
\hline & $\mathbf{a}$ & $\mathbf{b}$ & $\mathbf{c}$ & $\mathbf{d}$ & $\mathbf{e}$ & $\mathbf{f}$ \\
\hline NA & -1.16 & -0.78 & 1.62 & -0.38 & 1.09 & 1.05 \\
\hline ENP & 1.09 & -0.63 & -0.47 & 0.70 & -1.65 & 2.32 \\
\hline WNP & -0.22 & -0.84 & -0.90 & 1.26 & -0.95 & -0.19 \\
\hline
\end{tabular}

\section{Figures}



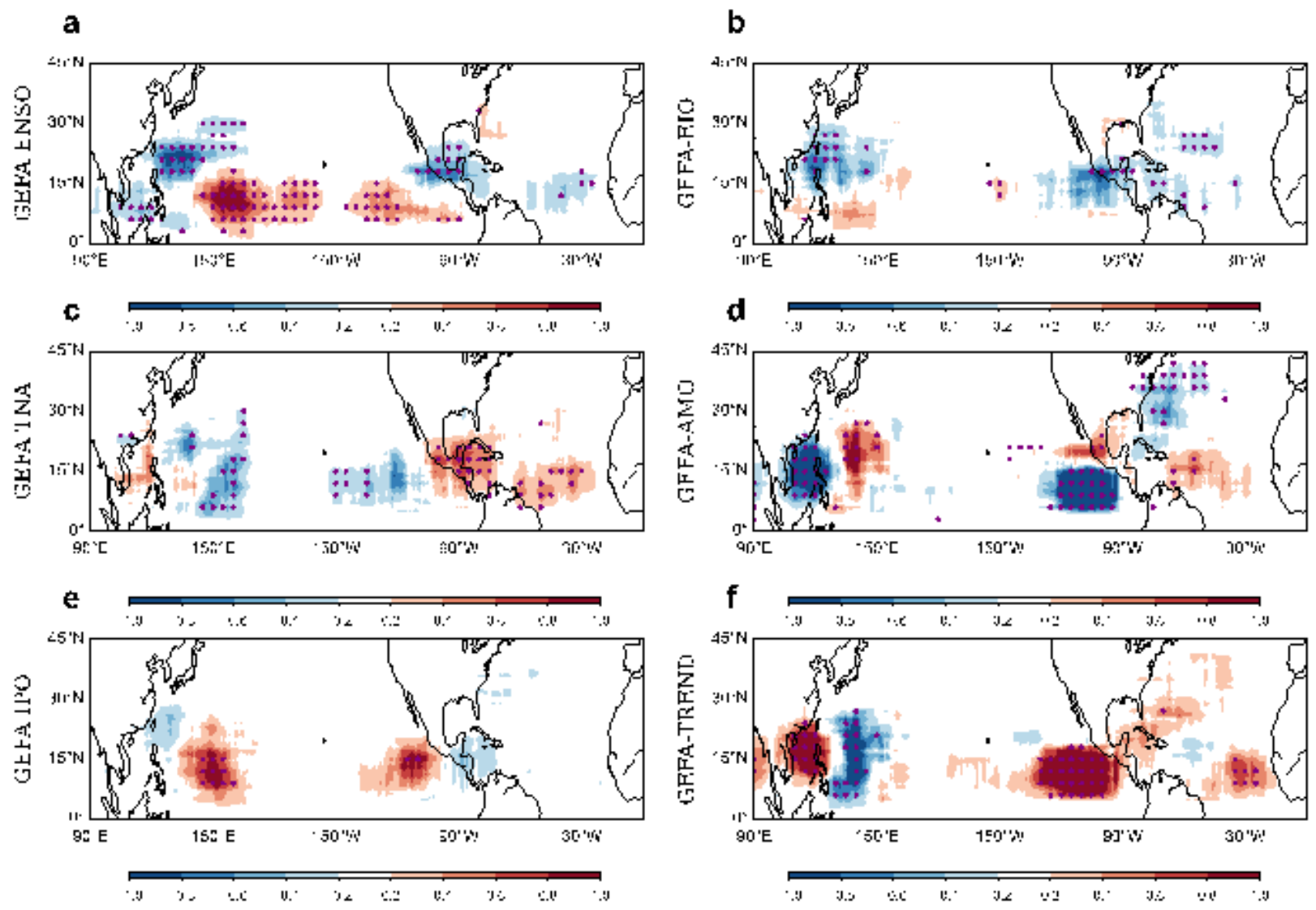

Figure 1

The feedback coefficients of the responses of TCGF in the NH during the TC season to the six key factors based on the GEFA. (a) ENSO, (b) EIO, (c) TNA, (d) AMO, (e) IPO, (f) GW. The dots represent the areas where the statistical significance is over $90 \%$ confidence level based on the Monte Carlo test with 100 random tests. 

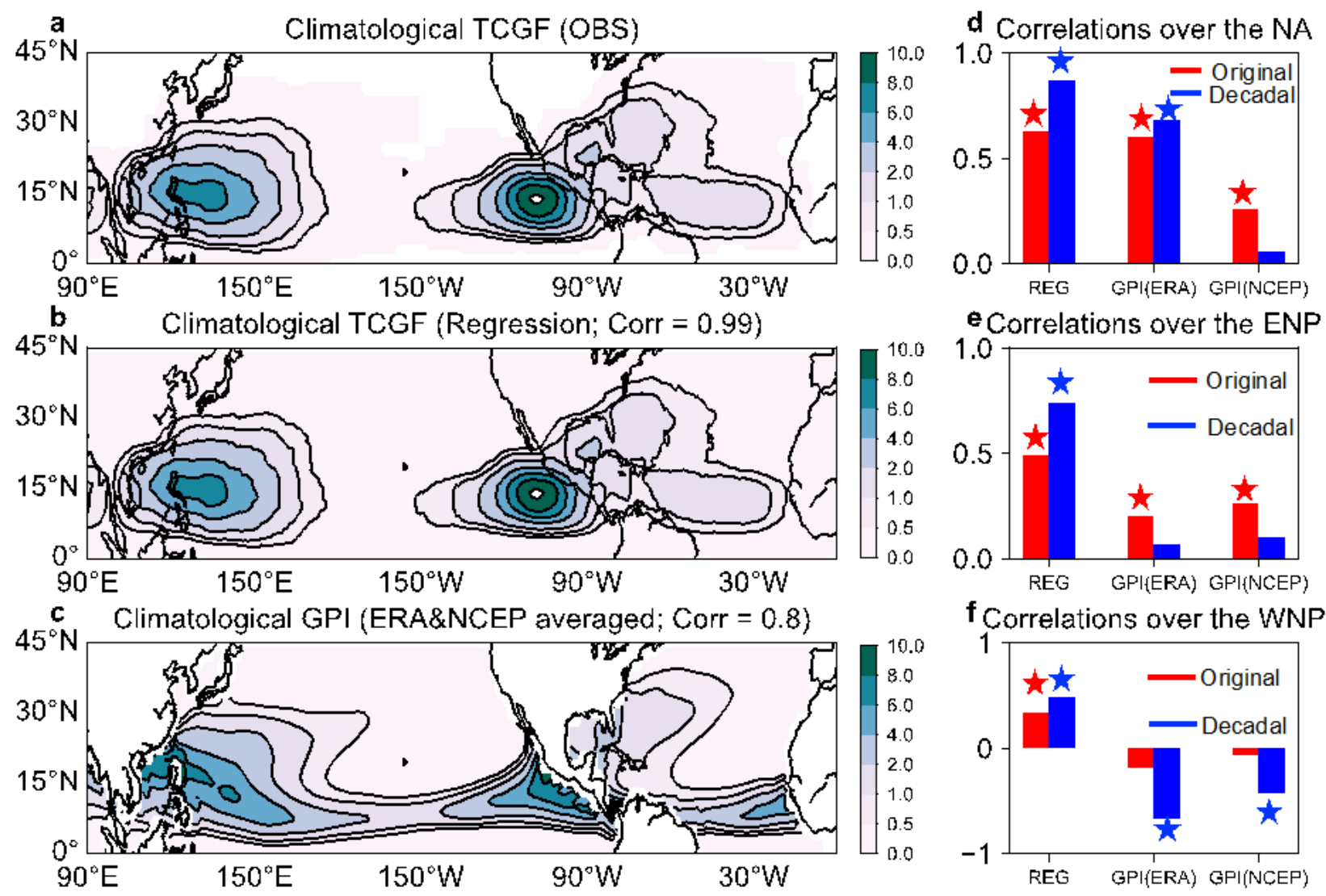

Figure 2

Comparisons of the observed and regressed climatological TCGF (or GPI) for the three main TC basins in the NH. (a) Climatological mean of the observed JJASON TCGF averaged during 1960-2019; (b) Climatological mean of the regressed TCGF and its correlation coefficient of spatial pattern with the observed TCGF; (c) Climatological mean of the commonly used GPI and its correlation coefficient of spatial pattern; (d) the correlation coefficients between observed and regressed TCGF using leave-out-one method (REG), GPI calculated based on ERA and NCEP reanalysis data (GPI/ERA and GPI/NCEP over the NA; (e) same as (d) but over the ENP; and (f) same as (d) but for the WNP. In (d-f), the red bars represent the correlation calculated using the original time series while the blue bars indicate the interannual variability of TCGF (GPI) filtered out using the fast Fourier transform; and the red and blue stars mean the correlations are significant above $95 \%$ confidence level based on Student's $t$ test with the adjusted degrees of freedom for the long-term variability. GPI are averaged in the main development regions of TCs, namely, $5-20^{\circ} \mathrm{N}, 30^{\circ}-70^{\circ} \mathrm{W}$ for the NA; $5^{\circ}-20^{\circ} \mathrm{N}, 90^{\circ} \mathrm{W}-130^{\circ} \mathrm{W}$ for the ENP; and $5^{\circ}-25^{\circ} \mathrm{N}, 110^{\circ}-160^{\circ} \mathrm{E}$ for the WNP. 

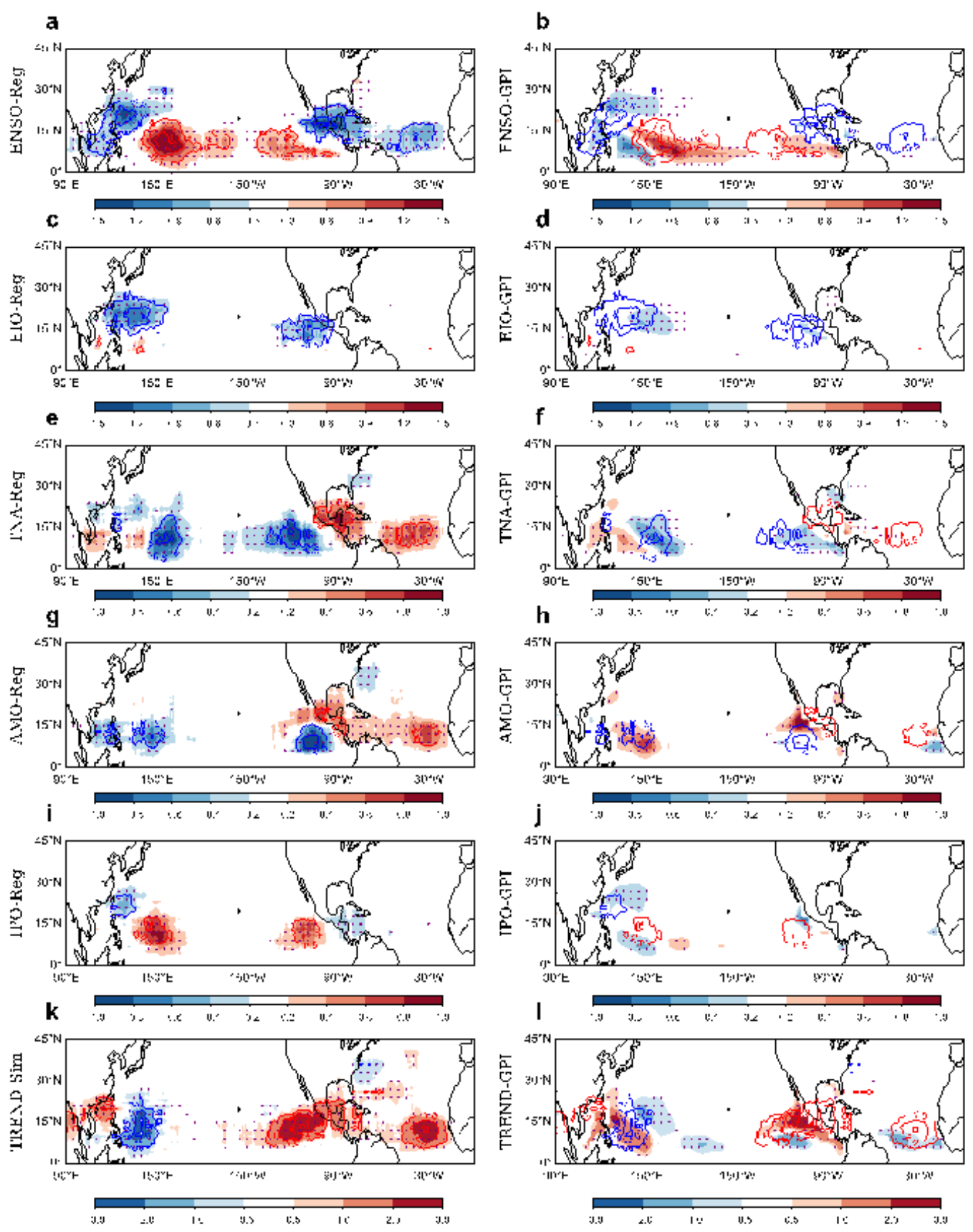

\section{Figure 3}

The connections of the observed TCGF, the multi-timescale regression model-estimated TCGF, and the commonly used GPI with the six key factors used in the multi-timescale regression model during 19602019. The composite differences of the observed (line), hindcast TCGFs (shaded) by (a) the multitimescale regression model and (b) the GPI between El Niño and La Niña years; by (c) the multi-timescale regression model and (d) the GPI between positive and negative EIO years; by (e) the multi-timescale 
regression model and ( $\mathrm{f}$ ) the GPI between positive and negative TNA years; by ( $\mathrm{g}$ ) the multi-timescale regression model and ( $h$ ) the GPI between positive and negative IPO years; by (i) the multi-timescale regression model and (j) the GPI between positive and negative AMO years; and the linear trend increments of the observed, hindcast TCGFs by ( $k$ ) the multi-timescale regression model and (l) the GPI. The dots indicate areas where the differences are statistically significant at the $95 \%$ confidence level by the Student's t test. The contours represent the observed TCGF and the shades stand for hindcast TCGFs by the multi-timescale regression model (left) and the GPI (right).

RCP2.6
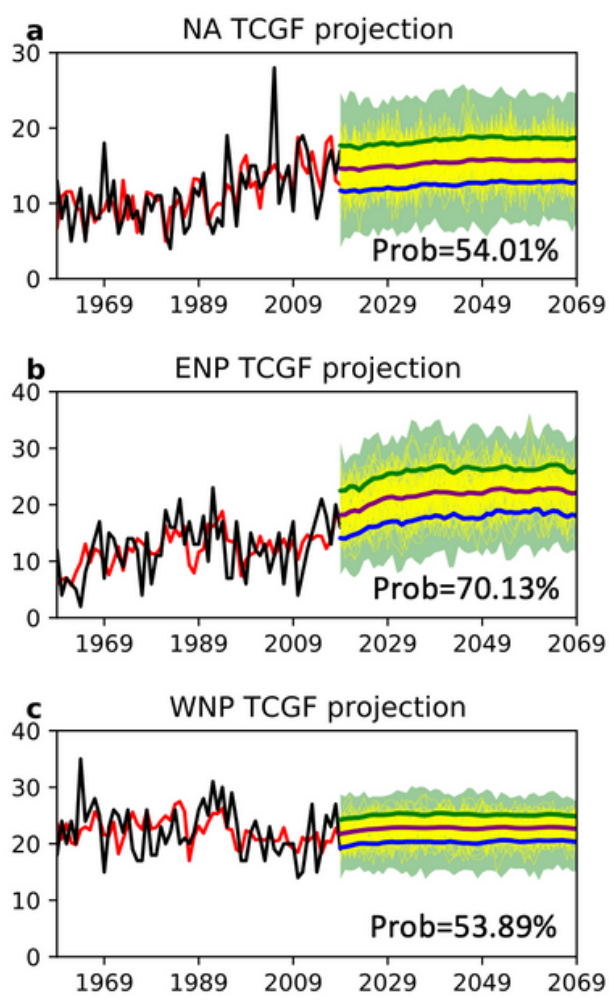

RCP4.5
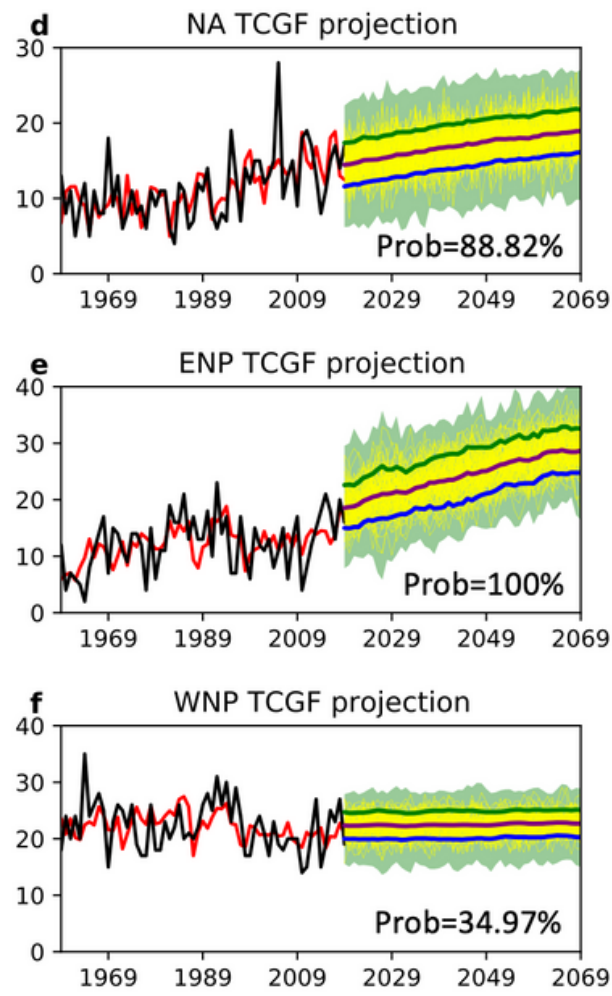

RCP8.5
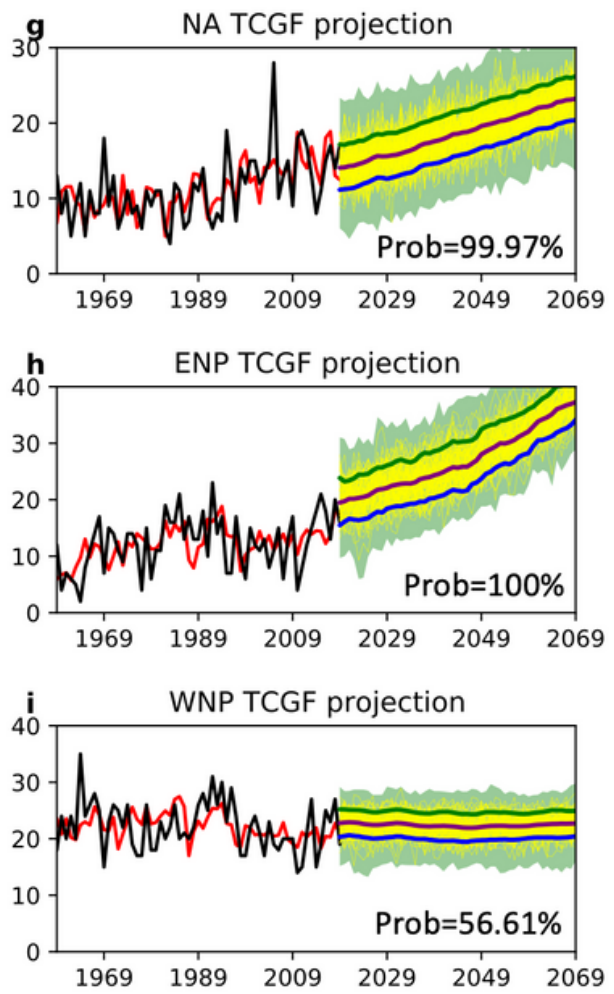

\section{Figure 4}

The projected TCGF changes in the three main TC basins in the NH from 2019 to 2069 under the RCP2.6, RCP4.5 and RCP8.5 scenarios. Here, the NH only includes NA ( $a, d$ and g), the ENP ( $b$, e and $h$ ) and the WNP (c, $f$ and i). (a-c) The RCP2.6 scenario, which means that the increasing SST anomalies and inter-/multi-decadal time series are derived from MPI-ESM under RCP2.6 forcing; (d-f) same as (a-c) but under the RCP4.5 scenario and ( $\mathrm{g}-\mathrm{i})$ same as (a-c) but under the RCP8.5 scenario. The inter-/multidecadal signals of the IPO and the AMO are from the second principal components of EOF analysis of SST in the respective Pacific and North Atlantic regions. And the global warming trend of SST are derived from the SST anomalies averaged over $45^{\circ} \mathrm{S}-45^{\circ} \mathrm{N}, 0^{\circ}-360^{\circ}$. The initial value (2019) is given based on observations. The shades represent the spread of the 100 ensemble members of time series from the MPI-ESM SST data, and the bold purple curves represent the ensemble mean of the 100 MPI-ESM members $\times 100$ cases/member while the green and blue represent, respectively, $10 \%$ and $90 \%$ percentiles. The yellow lines represent the projections for 100 random cases from 100 members and the shades indicate the spread. Here, the climatological mean TCGFs $(11.6,16.11,22.03$ for respective NA, ENP and 
WNP) from 1960-2019 are added to the multi-timescale regression model predicted TCGF anomalies in the plotted time series of TCGFs. Percentage on the lower right corner of each panel indicates the probability, which indicates the ratio of the ensemble members in which the trends are statistically significant at the $90 \%$ confidence level based on the Mann-Kendall test to the total (100) ensemble members.

\section{Supplementary Files}

This is a list of supplementary files associated with this preprint. Click to download.

- Supplementaryinformation.pdf 